\title{
Exploratory Compatibility Regularity of Traditional Chinese Medicine on Osteoarthritis Treatment: A Data Mining and Random Walk-Based Identification
}

\author{
Qiao Zhou $\mathbb{D}^{1,2}$ Jian Liu $\mathbb{D}^{1},{ }^{1,3}$ Ling Xin, ${ }^{3}$ Yanyan Fang $\mathbb{D}^{1},{ }^{1,3}$ Lei Wan, ${ }^{1,3}$ Dan Huang, ${ }^{1,3}$ \\ Jinchen Guo, ${ }^{1}$ Jianting Wen, ${ }^{1}$ and Bing Wang $\mathbb{C}^{4}$ \\ ${ }^{1}$ Institute of Rheumatism Prevention and Treatment of Traditional Chinese Medicine, \\ Anhui Academy of Chinese Medicine Sciences, Hefei 230031, China \\ ${ }^{2}$ Geriatrics Department, The Second Affiliated Hospital, Anhui University of Chinese Medicine, Hefei 230061, China \\ ${ }^{3}$ Department of Rheumatism Immunity, The First Affiliated Hospital, Anhui University of Chinese Medicine, Hefei 230031, China \\ ${ }^{4}$ School of Electrical \& Informatics Engineering, Anhui University of Technology, Ma'anshan 243002, China
}

Correspondence should be addressed to Jian Liu; liujianahzy@126.com and Bing Wang; wangbing@ustc.edu

Received 27 April 2021; Revised 19 October 2021; Accepted 29 October 2021; Published 22 November 2021

Academic Editor: Eliana Rodrigues

Copyright ( 2021 Qiao Zhou et al. This is an open access article distributed under the Creative Commons Attribution License, which permits unrestricted use, distribution, and reproduction in any medium, provided the original work is properly cited.

Osteoarthritis (OA) is a degressive and complex disease which is a growing public health problem on a global scale. On basis of an in-house database consisting of clinical records of 13,083 OA patients, the Traditional Chinese Medicine (TCM) was divided into 4 categories of medicines on the basis of the curative properties of herbs. Due to the lack of depth and internal relationship in the calculation results of TCM compatibility law data mining methods such as statistics and frequency analysis, we use a variety of multidimensional complex network methods that can efficaciously find the compatibility law of TCM, including similarity measure, graphical visualization of network diagram, random walking, and propensity score methods. We summarize common couplet medicines utilized for the treatment of osteoarthritis. The similarity measure method was used to investigate the commonly used drugs for the treatment of osteoarthritis. The method of association rule analysis is used to recognize the compatibility between the components. On basis of the propensity score methods, the evaluation displayed that, compared with single drug, the drug group increased ESR, CRP, C3, C4, IgG, and IgA more efficiently. Concluding, a random walk model was constructed to assess drug efficacy. After applying a random walk model, while revealing the compatibility among different components of TCM, their therapeutic efficacy against OA is analyzed. We obtained four groups of drug combination clusters by similarity measure and 11 pairs of highly connected drugs by association rules, which are cardinal drug combinations in the prescription for the treatment of OA. We also found that different traditional drug pairs were associated with different laboratory indexes, and drug combinations could better optimize laboratory indexes. This study presented that the TCM constituents complement one another. Besides, the therapeutic effects resulting from a variety of combinations of these constituents are quite different.

\section{Introduction}

As a chronic progressive joint disease, osteoarthritis inflicts harm to the physical and mental health of the middle-aged and the elderly patients [1]. Pathologically, OA is characterized by simultaneous catabolic and anabolic processes which cause changes in all joint tissues. Chondrogenic degeneration, ectopic bone formation, subchondral osteosclerosis, ligament and meniscus injuries, and synovial injury in the articular cavity are the main disease markers [2]. Most of the methods remedying OA of Western medicine have their special shortcomings [3]. TCM can prevent and treat osteoarthritis through multiple levels, multiple targets, and multiple ways by the use of holistic view. What is more, traditional Chinese medicine has proved its advantages in certain aspects such as reducing the 
side effects of drugs, reversing drug resistance, and enhancing the quality of life and survival rate of patients [4].

Chinese medicine prescription is the treatment of a certain disease by the clinician under the guidance of the theory of Chinese medicine through a reasonable combination of medicines to maximize the strengths and avoid weaknesses, adjust its bias, reduce its toxicity, enhance or change its original effect, and eliminate or alleviate its disadvantages to the human body. The pharmacological and pharmacodynamic relationship between herbs is considered to be the compatibility of Chinese medicine treatment. The law of compatibility of TCM is a key issue in the study of Chinese medicine prescription. It is the core aim of Chinese medicine prescription research to excavate the law of compatibility and clarify the scientific connotation of compatibility. As an efficacious method and significant technique to grope for traditional Chinese medicine, data mining method has formed a set of standardized research models and systems in the mining of compatibility law of TCM, which can better reveal the compatibility relationship between drugs [5].

This research is designed to explore the compatibility regularity and clinical therapeutic role of TCM in the treatment of osteoarthritis. We use a variety of data mining tools, such as cluster analysis, association rules, graphical visualization of network graphs, propensity scoring methods, and random walk models to analyze clinical OA data. The data mining method can analyze the frequency of herbs, the rule of formulation, and the change of formulation pattern obtained from the knowledge graph.

\section{Materials and Methods}

2.1. Materials. The inpatient data were collected for inpatients who were OA inpatients between July 2009 and March 2021 in the Department of Rheumatology and Immunology of the First Affiliated Hospital of Anhui University of Chinese Medicine. The dataset includes the use of Chinese herbal medicine, Huangqin Qingre Chubi capsules prescription preparation, Furong ointment, and disease-associated laboratory indices such as the inflammatory markers CRP and ESR and the immune indexes IgA, IgM, IgG, C3, and C4. The Ethics Committee of the First Affiliated Hospital of Anhui University of Chinese Medicine approved the study protocol. There were 13,776 patients with OA examined, including 13,083 with the treatment of Chinese herbal medicine. All patients were divided into a control group (Chinese herbal medicine alone) and an experimental group (Chinese herbal medicine plus Huangqin Qingre Chubi capsule/Furong ointment prescription preparation). The control group had 7,119 cases, and the experimental group had 5,964 cases.

\subsection{Methods}

2.2.1. Similarity Measure. We used Chinese herbal medicine as a variable, and we set it as 1 if used and 0 if not used. The systematic cluster analysis method is used to study the compatibility of Chinese herbal medicine by SPSS 22.0 (IBM
Corp., Armonk, NY, USA). We treat each herb as a point and calculate the distance between the points. The ones that are close to each other fall into one category, and the ones that are far away fall into another category. The similarity of Chinese herbs was calculated using the mean Euclidean measure [6]. Setting $x_{i}$ and $y_{i}(i=1,2, \ldots n)$ as the continuous points in the two-dimensional metric space of signal $x$ and signal $y$, the Euclidean distance of $\mathrm{x}$ and $y$ can be defined as follows [7]:

$$
d(x, y)=\sqrt{\sum_{i=1}^{n}\left(x_{i}-y_{i}\right)^{2}} .
$$

In the above equation, $x_{i}$ and $y_{i}$ are the $i$ th sampling data points of signal $x$ and signal $y$, respectively, and $n$ is the total number of sampling points. An analysis of the equation demonstrates that the higher the similarity between signal $x$ and signal $y$ is, the smaller the value of the Euclidean distance $d(x, y)$ is. On the contrary, the lower the similarity between signal $x$ and signal $y$ is, the larger the value of the Euclidean distance $d(x, y)$ is.

\subsubsection{Association Rules}

(1) Apriori Algorithm. 1 was used to indicate the application of Chinese herbal medicine or indicators, while no use was indicated by 0 . The interdependency of Chinese herbs medicine was identified by Apriori module in SPSS Clementine v.11.1 (IBM Corp., Armonk, NY, USA). We set the minimum support to $60 \%$, confidence to $80 \%$, and improvement to $>1$. Nowadays, the data mining technology of association rules is mainly on basis of Apriori algorithm, and its key optimization is to find all frequent itemsets in the trade database [8]. As a pattern of the form $X \longrightarrow Y$, an association rule means that the presence of the itemsets $X$ is associated with $Y$ in transactions [9]. The support degree, confidence degree, and Lift degree of an association rule between $\mathrm{X}$ and $Y$ are, respectively, as follows [10]:

$$
\begin{gathered}
\operatorname{Support}(X, Y)=P(X Y)=\frac{\text { number }(X Y)}{\text { num(AllSamples })}, \\
\text { Confidence }(X \Leftarrow Y)=P(X \mid Y)=\frac{P(X Y)}{P(Y)}, \\
\operatorname{Lift}(X \longrightarrow Y)=P(Y \mid X) \mid P(Y) .
\end{gathered}
$$

(2) Graphical Visualization of Network Diagram. We use the SPSS Clementine v.11.1 (IBM Corp., Armonk, NY, USA) "network" node to analyze Chinese medicine. Set the threshold to be absolute, strong links are thicker, and the maximum number of links that can be displayed is 80 . The upper limit of weak links is 35 , the lower limit of strong links is 100 , and the link size shows continuous changes. The thick, thin, and dashed lines indicate the strength of the links between drugs to generate an overall network diagram. 
2.2.3. Propensity Score Methods. Propensity score approaches, extensively applied to regulate for confounding in observational researches with dichotomous treatment modalities, mimic the intended roles of randomization by the balance of measured baseline covariates across treatment groups [11]. $Z$ means treatment allocation, $Y$ the continuous outcome, and the baseline covariates are displayed as $X=\left(X_{1}, \ldots, X_{p}\right)$; the propensity score is defined as [12]

$$
e(X)=P(Z=1 \mid X) .
$$

As the treatment index $\mathrm{Z}$ is binary and it is supposed that the logistic regression is parametrized by $\alpha=\left(\alpha_{0}, \alpha_{1}, \ldots\right.$, $\left.\alpha_{p}\right)^{\top}$,

$$
\log \left\{\frac{e(X))}{(1-e(X))}\right\}-X^{\top} \alpha .
$$

For every participant indexed by subscript $i$, it is possible to estimate a probability in the treatment or control arm, given the baseline features from the fitted propensity score model as

$$
\widehat{e}_{i}=\widehat{e}\left(X_{i}\right)=\frac{\exp \left(X_{i}^{\top} \widehat{\alpha}\right)}{1+\exp \left(X_{i}^{\top} \widehat{\alpha}\right)} .
$$

The schematic diagram of propensity matching is shown in Figure 1.

2.2.4. Random Walking. The evaluation of the laboratory indices random walking model is realized through the ORACLE $10 \mathrm{~g}$ tool.

Random walk was first put forward by Pearson in 1905 [13]. The walk upward movement is $(u(i)=+1)$ and the downward movement is down $(u(i)=-1)$ by one-unit length ( $u$ ) for each step $i$. Consequently, the random walk ultimately stimulates the quantification of this correlation by calculating the "net displacement" $(y)$ of the walker after one step, which is the sum of the unit steps $u(i) f$ of each step $i[14]$ :

$$
\gamma(l)=\sum_{i=1}^{1} u(i) .
$$

The root mean square fluctuation $F(l)$ about the average of the displacement is an important statistical quantity characteristic. $F(l)$ is defined as the difference between the average of the square and the square of the average,

$$
F^{2}(l)=\overline{\left[\Delta \gamma(l)-\overline{\Delta y(l)]^{2}}\right.}=\overline{[\Delta y(l)]^{2}}-[\overline{\Delta y(l)}]^{2},
$$

of a quantity $\Delta y(l)$ defined by

$$
\Delta \gamma(l)=\gamma\left(l_{0}+l\right)-\gamma\left(l_{0}\right) .
$$

We can comprehend that equation (1) walks a set of calipers with a fixed distance gauge, equation (2) sequentially moves the starting point from $l_{0}=1$ to $l_{0}=2$ and so on, equation (6) calculates the quantity $\Delta y(l)$ and its square for each $l_{0}$, and equations (7a) and (7b) average all calculated quantities to obtain the following equation:

$$
F^{2}(l) \sim l^{a}, \quad \text { with } \alpha \neq \frac{1}{2}
$$

2.3. Statistical Processing. The analysis of all data was made by SPSS v.22.0 (IBM Corp., Armonk, NY, USA). The operation of a nonparametric test on two associated samples was made for the control and experimental groups before and after treatment. Variations in characteristics between groups were analyzed using the Mann-Whitney rank-sum test or $\chi^{2}$ tests. Variations were recognized to be statistically significant at $P<0.05$.

\section{Result}

3.1. Administration of Chinese Herbal Medicine in $O A$ Treatment. There were 13,776 patients with OA researched, among whom 13,083 were cured with Chinese herbal medicine. Every OA patient took 1 prescription of Chinese herbal medicine with $>420$ kinds. The first 20 Chinese herbal medicines were separated into 4 categories in the light of their potent effect: Poria, Radix Glycyrrhizae, Pericarpium Citri Reticulatae, Semen Coicis, and Rhizoma Dioscoreae Oppositae to invigorate spleen for eliminating dampness; Flos Carthami, Radix Salviae Miltiorrhizae, Semen Persicae, Caulis Spatholobi, and Radix Achyranthis Bidentatae to promote blood circulation to remove meridian obstruction; Radix et Rhizoma Clematidis Chinensis, Herba Siegesbeckiae, Radix Angelicae Biserratae, Herba Gaultheriae Grenulatae, and Herba Lycopodii Japonici to dispel wind and eliminate dampness; and Herba Taraxaci Mongolici, Herba Hedyotis, Plantago asiatica L., Cortex Phellodendri Amurensis, and Radix Scutellariae Baicalensis to clear away heat and toxic materials. On the basis of five flavors, sweet taste is supervised up to a maximum of 59,458 times, while bitter taste is given at the most 69,650 times. According to tropism of taste of Chinese herbal medicine, there are 44,470 uses of Chinese herbal medicine belonging to the spleen meridian maximally (Table 1).

3.2. Similarity Measure of Chinese Herbal Medicine in the Treatment of Osteoarthritis. The systematic clustering method for cluster analysis of 20 traditional Chinese medicines for treating osteoarthritis is adopted. When the Euclidean distance is 15, the following four groups of drug combination clusters can be obtained (the other 2 drugs are invalid clusters) (Figure 2).

Set1: Flos Carthami, Radix Salviae Miltiorrhizae, Poria, Radix Glycyrrhizae, Semen Coicis, Pericarpium Citri Reticulatae, Rhizoma Dioscoreae Oppositae, and Semen Persicae.

Set2: Herba Gaultheriae Grenulatae, Herba Lycopodii Japonici, Radix Angelicae Biserratae, and Caulis Spatholobi.

Set3: Plantago asiatica L., Cortex Phellodendri Amurensis, and Radix Scutellariae Baicalensis.

Set4: Herba Taraxaci Mongolici, Herba Hedyotis, and Herba Siegesbeckiae. 


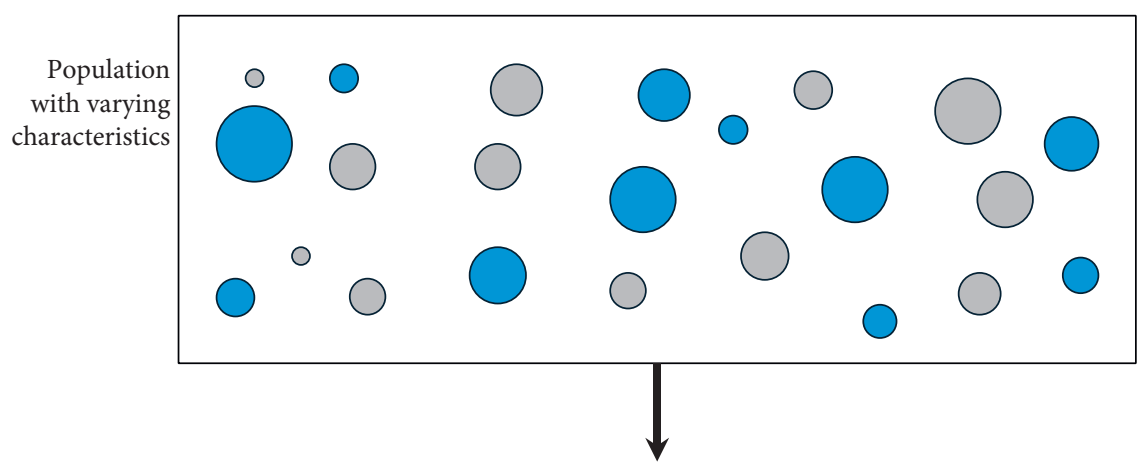

Study Group with Matching

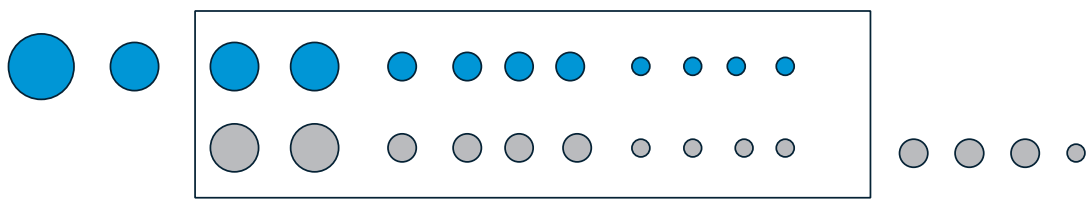

Experimental group

Control group

Figure 1: Diagrammatic sketch of propensity score methods.

Table 1: Application of Chinese herbal medicine in OA treatment.

\begin{tabular}{|c|c|c|c|c|}
\hline Category & Herb & Number & Nature and taste & Meridian tropism \\
\hline \multirow{5}{*}{ Invigorating spleen for eliminating dampness } & Poria & 10376 & Sweet, flat, light & \multirow{2}{*}{$\begin{array}{l}\text { Spleen, kidney } \\
\text { Heart, lung, stomach, } \\
\text { spleen }\end{array}$} \\
\hline & Radix Glycyrrhizae & 9789 & Gump, flat & \\
\hline & Pericarpium Citri Reticulatae & 9625 & $\begin{array}{l}\text { Pungent, warm, } \\
\text { bitter }\end{array}$ & Spleen \\
\hline & Semen Coicis & 9044 & Sweet, light & Spleen, stomach \\
\hline & Rhizoma Dioscoreae Oppositae & 8590 & Sweet, flat & Spleen, lung, kidney \\
\hline \multirow{5}{*}{$\begin{array}{l}\text { Removing meridian obstruction by promoting } \\
\text { blood circulation }\end{array}$} & Flos Carthami & 10100 & \multirow{5}{*}{$\begin{array}{c}\text { Pungent, warm } \\
\text { Bitter, slightly cold } \\
\text { Bitter, flat, sweet } \\
\text { Bitter, sweet, warm } \\
\text { Bitter, sweet, sour, } \\
\text { flat }\end{array}$} & Heart, liver \\
\hline & Radix Salviae Miltiorrhizae & 9769 & & Heart, liver \\
\hline & Semen Persicae & 8593 & & Heart, liver \\
\hline & Caulis Spatholobi & 6622 & & Liver, kidney \\
\hline & Radix Achyranthis Bidentatae & 4284 & & Liver, kidney \\
\hline \multirow{5}{*}{ Dispelling wind and eliminating dampness } & $\begin{array}{c}\text { Radix et Rhizoma Clematidis } \\
\text { Chinensis }\end{array}$ & 7743 & Pungent, salt, warm & Bladder \\
\hline & Herba Siegesbeckiae & 5511 & $\begin{array}{l}\text { Bitter, bitter, slightly } \\
\text { cold }\end{array}$ & Liver, kidney \\
\hline & Radix Angelicae Biserratae & 4395 & $\begin{array}{l}\text { Pungent, bitter, } \\
\text { warm }\end{array}$ & Kidney, bladder \\
\hline & Herba Gaultheriae Grenulatae & 4127 & Sweet, pungent & Lung, liver \\
\hline & Herba Lycopodii Japonici & 3606 & $\begin{array}{l}\text { Bitter, pungent, } \\
\text { warm }\end{array}$ & Liver, spleen, kidney \\
\hline \multirow{5}{*}{ Eliminating heat and toxic materials } & Herba Taraxaci Mongolici & 7288 & $\begin{array}{l}\text { Bitter, slightly cold, } \\
\text { sweet }\end{array}$ & \multirow{5}{*}{$\begin{array}{c}\text { Liver, stomach } \\
\text { Stomach, larger } \\
\text { intestine } \\
\text { Liver, kidney, lung } \\
\text { Kidney, bladder } \\
\text { Lung, gallbladder, } \\
\text { spleen }\end{array}$} \\
\hline & Herba Hedyotis & 4661 & $\begin{array}{l}\text { Bitter, slightly cold, } \\
\text { sweet }\end{array}$ & \\
\hline & Plantago asiatica L. & 3034 & Sweet, slightly cold & \\
\hline & Cortex Phellodendri Amurensis & 2832 & Bitter & \\
\hline & Radix Scutellariae Baicalensis & 2464 & Bitter & \\
\hline
\end{tabular}

3.3. Analysis of Association Rules of Traditional Chinese Medicine or Immune-Inflammatory Indices in the Treatment of Osteoarthritis. The compatibility of the two TCM is to increase efficiency and reduce toxicity. The compatibility mode of the prescription is further tested. We analyze the correlation degree of core Chinese medicine, where the 


FlosCarthami
SemenPersicae
RadixSalviaeMiltiorrhizae
Poria
PericarpiumCitriReticulatae
RadixGlycyrrhizae
SemenCoicis
RhizomaDioscoreaeOppositae
RadixetRhizomaClematidisChinensis
HerbaGaultheriaeGrenulatae
HerbaLycopodiijaponici
RadixAngelicaeBiserratae
CaulisSpatholobi
RadixAchyranthisBidentatae
PlantagoasiaticaL
CortexPhellodendriAmurensis
RadixScutellariaeBaicalensis
HerbaTaraxaciMongolici
HerbaHedyotdis
HerbaSiegesbeckiae

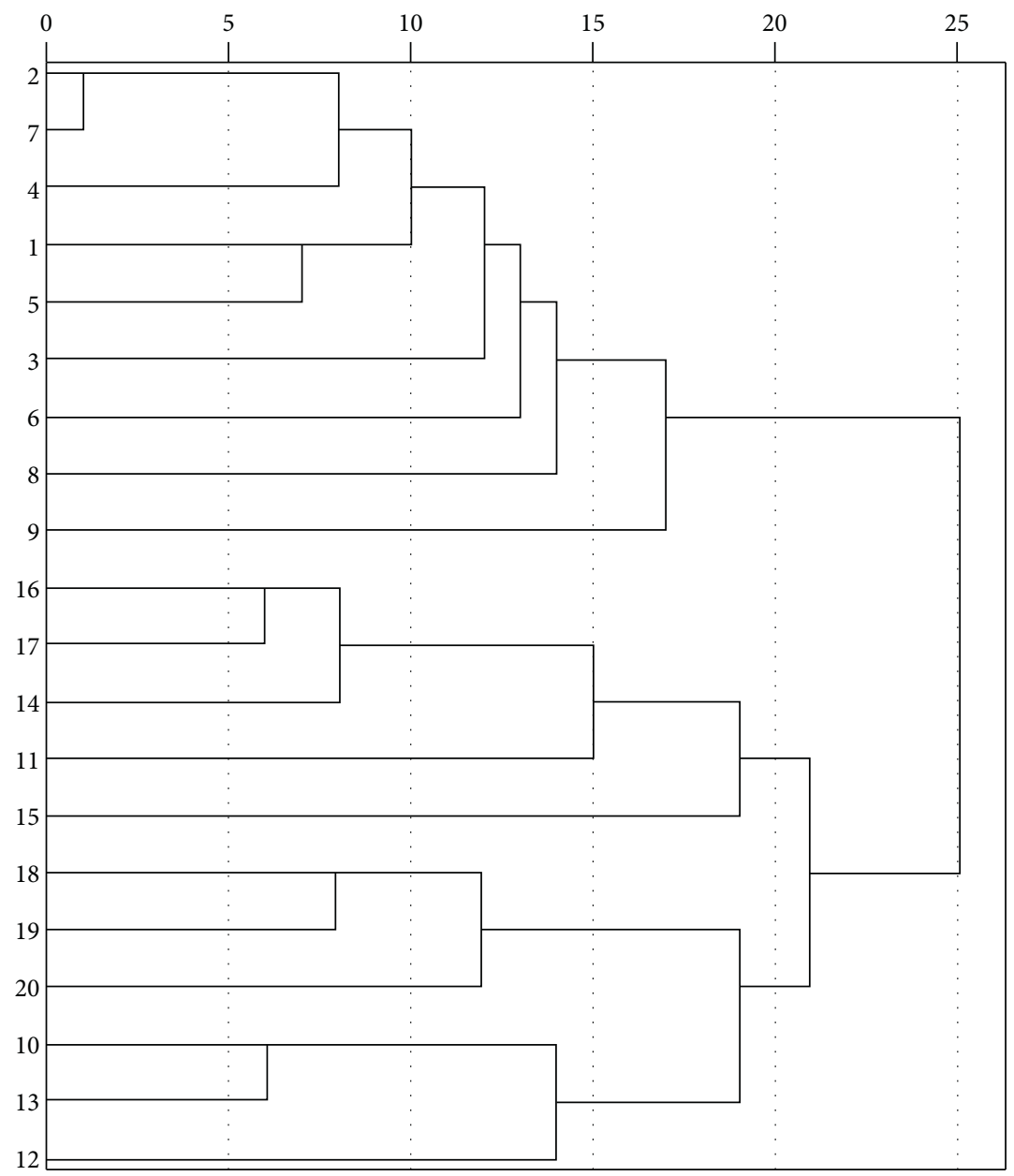

Figure 2: Similarity measure of Chinese herbal medicine treatment of osteoarthritis. Note: Euclidean distance $=15$ o'clock; Chinese medicine is separated into four groups. Neither Radix et Rhizoma Clematidis Chinensis nor Radix Achyranthis Bidentatae is included in other taxa.

minimum support is set to $70 \%$, and the minimum confidence is set to $80 \%$. The lift is $>1, P \leq 0.001$. According to the analysis results of association rules, the higher the promotion (gain), the stronger the correlation. We define each traditional Chinese medicine as an itemset. Finally, we secured 11 pairs of highly connected drugs. They are cardinal drug combinations in the prescription for the treatment of OA. The improvement degree of all drug combinations is greater than 1.0, indicating that these drug combinations are statistically significant (Table 2 ). In addition, by constructing an association network diagram of high-frequency drugs, it clearly and intuitively reflects the degree of association between drugs. Herba Taraxaci Mongolici, Herba Hedyotis, Flos Carthami, Caulis Spatholobi, Semen Persicae, Semen Coicis, Rhizoma Dioscoreae Oppositae, Radix Salviae Miltiorrhizae, Radix Achyranthis Bidentatae, Radix Glycyrrhizae, and Poria have strong correlation in node degree. They often appear in pairs and are the core drug combination (Figure 3). Simultaneously, the minimum support is set to $60 \%$ and the minimum confidence to $80 \%$; after the analysis of the Apriori module, each item is ranked with the highest confidence level. We conduct correlation analysis on drugs and immune-inflammatory indices to analyze the relationship between immune-inflammatory indices optimization and Chinese herbs compatibility. We found that Herba Taraxaci Mongolici, Flos Carthami, and Semen Coicis are related to inflammatory index, such as ESR and CRP. Semen Persicae, Poria, Radix Glycyrrhizae, and Radix Salviae Miltiorrhizae are related to immune index, for instance, IgM, IgA, and IgG. Radix Glycyrrhizae and Pericarpium Citri Reticulatae are bounded up with $\mathrm{C} 4$ and C3. The improvement degree of the above correlation results is $>1$ (Table 3).

3.4. Improvement of Immune-Inflammatory Indices. First, we assembled one-to-one propensity score methods matching the clinical background between the two groups to minimize the imbalance from measured baseline covariates. The matched groups were balanced by age, sex, BMI, length of stay, and underlying diseases (coronary heart disease, hypertension, cerebral infarction, diabetes, chronic gastritis, anemia, osteoporosis, and fatty liver) (Table 4). One-to-one nearest neighbour caliper matching was used to match 
TABLe 2: Association of Chinese herbal medicine in OA treatment.

\begin{tabular}{|c|c|c|c|c|}
\hline Items (LHS $\Rightarrow$ RHS) & Support (\%) & Confidence (\%) & Lift & $P$ value \\
\hline$\{$ Pericarpium Citri Reticulatae $\} \Rightarrow\{$ Poria $\}$ & 73.57 & 87.71 & 1.11 & $\leq 0.001$ \\
\hline$\{$ Radix Salviae Miltiorrhizae $\} \Rightarrow\{$ Flos Carthami $\}$ & 74.67 & 85.38 & 1.11 & $\leq 0.001$ \\
\hline$\{$ Radix Salviae Miltiorrhizae $\} \Rightarrow\{$ Poria $\}$ & 74.67 & 85.02 & 1.07 & $\leq 0.001$ \\
\hline$\{$ Flos Carthami $\} \Rightarrow\{$ Semen Persicae $\}$ & 77.20 & 83.93 & 1.28 & $\leq 0.001$ \\
\hline$\{$ Radix Glycyrrhizae $\} \Rightarrow\{$ Poria $\}$ & 74.82 & 83.69 & 1.05 & $\leq 0.001$ \\
\hline$\{$ Pericarpium Citri Reticulatae $\} \Rightarrow\{$ Flos Carthami $\}$ & 73.56 & 83.28 & 1.08 & $\leq 0.001$ \\
\hline$\{$ Flos Carthami $\} \Rightarrow\{$ Poria $\}$ & 77.20 & 82.87 & 1.04 & $\leq 0.001$ \\
\hline$\{$ Pericarpium Citri Reticulatae $\} \Rightarrow\{$ Radix Salviae Miltiorrhizae $\}$ & 73.57 & 82.75 & 1.11 & $\leq 0.001$ \\
\hline$\{$ Flos Carthami $\} \Rightarrow\{$ Radix Salviae Miltiorrhizae $\}$ & 77.20 & 82.58 & 1.11 & $\leq 0.001$ \\
\hline$\{$ Radix Salviae Miltiorrhizae $\} \Rightarrow\{$ Pericarpium Citri Reticulatae $\}$ & 74.67 & 81.53 & 1.11 & $\leq 0.001$ \\
\hline$\{$ Poria $\} \Rightarrow\{$ Pericarpium Citri Reticulatae $\}$ & 79.31 & 81.36 & 1.11 & $\leq 0.001$ \\
\hline
\end{tabular}

Values are \% degrees of relevancy.

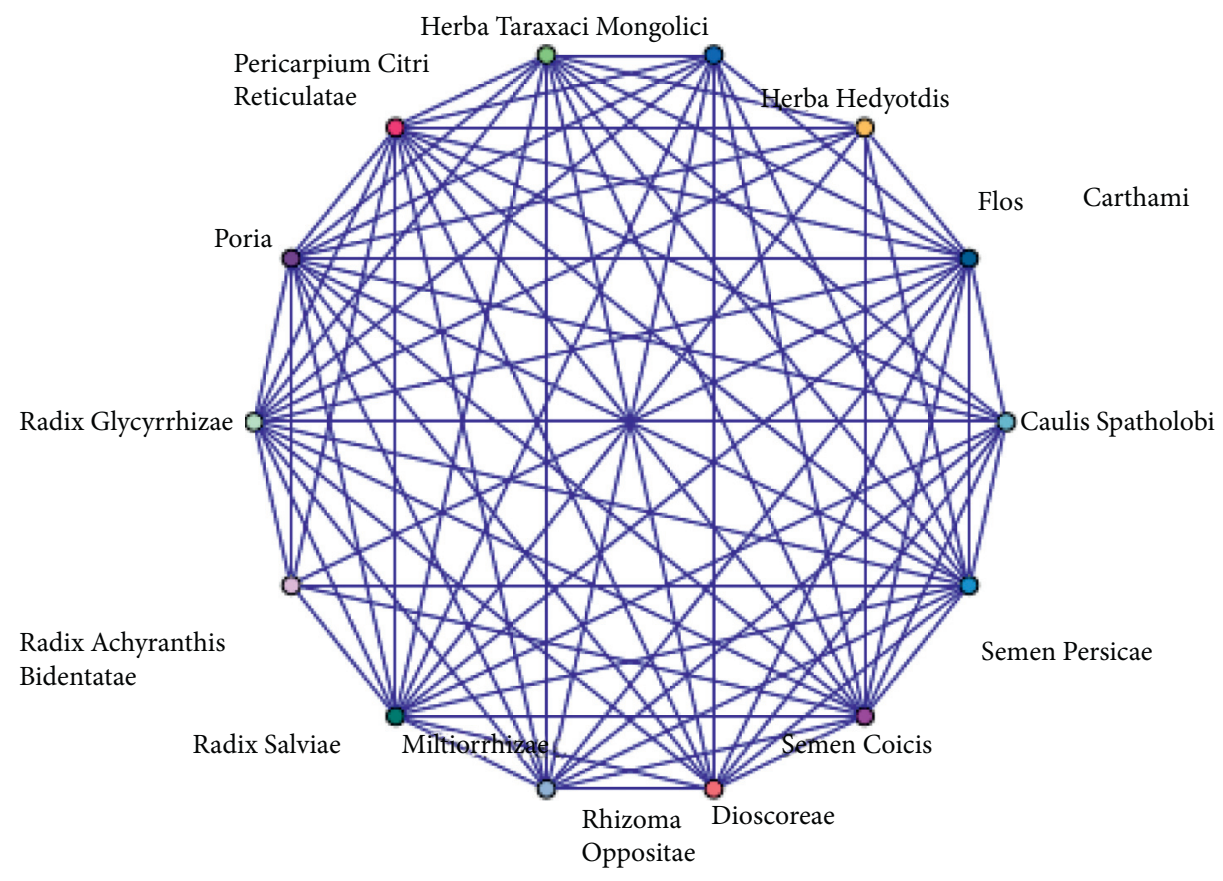

FIGURE 3: Graphical visualization of network diagram of Chinese herbal medicine in OA treatment.

TABLE 3: Association of Chinese herbal medicine and immune-inflammatory indices in OA treatment.

\begin{tabular}{|c|c|c|c|c|}
\hline Items (LHS $\Rightarrow$ RHS) & Support & Confidence & Lift & $P$ value \\
\hline$\{$ Herba Taraxaci Mongolici and Flos Carthami $\} \Rightarrow\{$ ESR $\}$ & 60.52 & 82.86 & 1.02 & $\leq 0.001$ \\
\hline$\{$ Herba Taraxaci Mongolici and Semen Coicis $\} \Rightarrow\{$ CRP $\}$ & 61.64 & 87.46 & 1.02 & $\leq 0.001$ \\
\hline$\{$ Semen Persicae and Poria $\} \Rightarrow\{$ IgA $\}$ & 61.82 & 83.50 & 1.02 & $\leq 0.001$ \\
\hline$\{$ Radix Glycyrrhizae and Poria $\} \Rightarrow\{$ IgM $\}$ & 64.81 & 75.25 & 1.03 & $\leq 0.001$ \\
\hline$\{$ Radix Glycyrrhizae and Radix Salviae Miltiorrhizae $\} \Rightarrow\{\operatorname{IgG}\}$ & 63.11 & 99.89 & 1.00 & $\leq 0.001$ \\
\hline$\{$ Radix Glycyrrhizae and Pericarpium Citri Reticulatae $\} \Rightarrow\{\mathrm{C} 3\}$ & 62.32 & 85.85 & 1.01 & $\leq 0.001$ \\
\hline$\{$ Radix Glycyrrhizae and Pericarpium Citri Reticulatae $\} \Rightarrow\{\mathrm{C} 4\}$ & 68.73 & 89.82 & 1.00 & $\leq 0.001$ \\
\hline
\end{tabular}

Values are \% degrees of relevancy.

patients based on the propensity score using a caliper equal to 0.2 of the SD of the logit of the propensity score. The age, sex, BMI, LOS, and underlying diseases (coronary heart disease, hypertension, cerebral infarction, diabetes, and osteoporosis) were significantly different between the experimental group and the control group but were comparable between the matched experimental group and the matched control group after propensity score matching. The immune-inflammatory indices between the matched experimental group and the matched control group were also 
TABLE 4: Baseline characteristics of all patients.

\begin{tabular}{|c|c|c|c|c|c|c|c|}
\hline \multirow{2}{*}{\multicolumn{2}{|c|}{ Variables }} & \multicolumn{3}{|c|}{ Before matching } & \multicolumn{3}{|c|}{ After matching } \\
\hline & & $\begin{array}{l}\text { Control group } \\
(n=7,119)\end{array}$ & $\begin{array}{c}\text { Experimental } \\
\text { group }(n=5,964)\end{array}$ & $\begin{array}{c}P \\
\text { value }\end{array}$ & $\begin{array}{c}\text { Control group } \\
\quad(n=4,438)\end{array}$ & $\begin{array}{c}\text { Experimental } \\
\text { group }(n=4,438)\end{array}$ & $P$ value \\
\hline Demographics & $\begin{array}{l}\text { Age/year, } \\
\text { mean } \pm S D\end{array}$ & $58.56 \pm 13.71$ & $62.75 \pm 12.88$ & $<0.001$ & $60.06 \pm 13.84$ & $59.84 \pm 12.82$ & 0.068 \\
\hline \multirow{2}{*}{ Sex } & Male, $n(\%)$ & $1,402(19.69)$ & $1,582(26.53)$ & $<0.001$ & $907(20.43)$ & $912(20.55)$ & 0.072 \\
\hline & Female, $n(\%)$ & $5,717(80.31)$ & $4,382(73.47)$ & $<0.001$ & $3,531(79.56)$ & $3,526(79.45)$ & 0.080 \\
\hline $\begin{array}{l}\text { Anthropometric } \\
\text { measurements }\end{array}$ & BMI, $\mathrm{kg} / \mathrm{m}^{2}$ & $28.36 \pm 5.60$ & $26.56 \pm 6.50$ & $<0.001$ & $27.12 \pm 6.25$ & $27.68 \pm 6.04$ & 0.094 \\
\hline $\begin{array}{l}\text { Clinical } \\
\text { characteristics }\end{array}$ & $\begin{array}{l}\text { LOS, days, } \\
\text { mean } \pm \text { SD }\end{array}$ & $15.05 \pm 7.77$ & $17.10 \pm 9.08$ & $<0.001$ & $16.36 \pm 8.36$ & $16.49 \pm 7.91$ & 0.074 \\
\hline \multirow{8}{*}{ Underlying diseases } & $\begin{array}{l}\text { Coronary heart } \\
\text { disease, } n(\%)\end{array}$ & $354(4.97)$ & $233(3.91)$ & $<0.001$ & $189(4.26)$ & $183(4.12)$ & 0.061 \\
\hline & $\begin{array}{l}\text { Hypertension, } n \\
(\%)\end{array}$ & $193(2.71)$ & $1,243(20.84)$ & $<0.001$ & $193(4.34)$ & $199(4.48)$ & 0.056 \\
\hline & $\begin{array}{c}\text { Cerebral } \\
\text { infarction, } n(\%)\end{array}$ & $236(3.32)$ & $768(12.88)$ & $<0.001$ & $229(5.16)$ & $231(5.21)$ & 0.063 \\
\hline & Diabetes, $n(\%)$ & $119(1.67)$ & $610(10.23)$ & $<0.001$ & $119(2.68)$ & $132(2.97)$ & 0.051 \\
\hline & $\begin{array}{c}\text { Chronic gastritis, } n \\
(\%)\end{array}$ & $1,353(19.01)$ & $744(12.47)$ & $<0.001$ & $572(12.89)$ & $619(13.95)$ & $<0.001$ \\
\hline & Anemia, $n(\%)$ & $207(2.91)$ & $167(2.80)$ & $<0.001$ & $93(2.10)$ & $133(3.00)$ & $<0.001$ \\
\hline & $\begin{array}{c}\text { Osteoporosis, } n \\
\text { (\%) }\end{array}$ & $1,861(26.14)$ & $1,616(27.10)$ & $<0.001$ & $1,214(27.35)$ & $1,202(27.08)$ & 0.063 \\
\hline & Fatty liver, $n(\%)$ & $768(10.79)$ & $700(11.74)$ & $<0.001$ & $361(8.13)$ & $475(10.70)$ & $<0.001$ \\
\hline \multirow{7}{*}{ Index } & $\operatorname{ESR}(\mathrm{mm} / \mathrm{h})$ & $28.79 \pm 15.89$ & $14.44 \pm 11.97$ & $<0.001$ & $30.18 \pm 16.92$ & $29.26 \pm 12.84$ & 0.112 \\
\hline & $\mathrm{CRP}(\mathrm{mg} / \mathrm{L})$ & $14.44 \pm 7.96$ & $27.80 \pm 9.26$ & $<0.001$ & $14.66 \pm 8.48$ & $16.50 \pm 7.12$ & 0.124 \\
\hline & $\operatorname{IgA}(\mathrm{g} / \mathrm{L})$ & $2.35 \pm 1.07$ & $2.65 \pm 1.25$ & $<0.001$ & $2.38 \pm 1.09$ & $2.41 \pm 1.21$ & 0.085 \\
\hline & $\operatorname{IgM}(g / L)$ & $1.26 \pm 0.67$ & $1.23 \pm 0.72$ & 0.427 & $1.20 \pm 0.63$ & $1.21 \pm 0.70$ & 0.354 \\
\hline & $\operatorname{IgG}(\mathrm{g} / \mathrm{L})$ & $12.40 \pm 3.99$ & $15.91 \pm 4.06$ & $<0.001$ & $12.61 \pm 4.04$ & $13.04 \pm 4.14$ & 0.214 \\
\hline & $\mathrm{C} 3(\mathrm{~g} / \mathrm{L})$ & $83.69 \pm 49.72$ & $93.12 \pm 53.61$ & $<0.001$ & $97.16 \pm 39.22$ & $90.01 \pm 54.33$ & 0.359 \\
\hline & $\mathrm{C} 4(\mathrm{~g} / \mathrm{L})$ & $26.04 \pm 13.44$ & $22.27 \pm 14.53$ & $<0.001$ & $23.43 \pm 11.69$ & $22.50 \pm 14.69$ & 0.212 \\
\hline
\end{tabular}

Note: BMI, body mass index; LOS, length of hospital stay; ESR: erythrocyte sedimentation rate; CRP: C-reactive protein; IgA: immunoglobulin A; IgM: immunoglobulin M; IgG: immunoglobulin G; C3: complement C3; C4: complement C4.

comparable. After that, by comparison with those before treatment, ESR, IgA, IgG, CRP, IgM, C3, and C4 were reduced greatly in both groups after treatment. After treatment, ESR, IgA, CRP, IgG, C3, and C4 reduced greatly in the experimental group compared to the control group (Table 5).

\subsection{Evaluation of Immune-Inflammatory Indices by Random} Walking Model. The ESR of the control group had a total of 6253 comprehensive evaluation records. The clinical significance is that, for every increase in the integrated index of the patient, 9.26 steps need to be walked, or, every step forward, the comprehensive improvement rate is $22.60 \%$. There are a total of 6883 comprehensive evaluation records of ESR in the experimental group. The clinical significance is that, for every increase in the integrated index of the patient, 5.11 steps need to be walked, or, every step forward, the integrated improvement rate is $36.20 \%$. There were 6,933 and 7,603 integrated evaluation records for CRP in both groups. The patient improvement indexes in both groups were 0.276 and 0.426 , respectively. With regard to the clinical significance, for every increase in the integrated index, the patients had to walk 7.28 and 4.18 steps. There are
TABLE 5: Improvement of immune-inflammatory indices.

\begin{tabular}{lccccc}
\hline \multirow{2}{*}{ Index } & \multicolumn{2}{c}{ Control group } & \multicolumn{3}{c}{ Experimental group } \\
& $Z_{0}$ & $P_{0}$ value & $Z_{1}$ & $P_{1}$ value & $P_{2}$ value \\
\hline ESR $(\mathrm{mm} / \mathrm{h})$ & -25.59 & $\leq 0.001$ & -37.85 & $\leq 0.001$ & $\leq 0.001$ \\
CRP $(\mathrm{mg} / \mathrm{L})$ & -33.98 & $\leq 0.001$ & -48.04 & $\leq 0.001$ & $\leq 0.001$ \\
IgA $(\mathrm{g} / \mathrm{L})$ & -14.54 & $\leq 0.012$ & -19.10 & $\leq 0.001$ & $\leq 0.001$ \\
IgM $(\mathrm{g} / \mathrm{L})$ & -2.50 & $\leq 0.001$ & -5.25 & $\leq 0.001$ & 0.242 \\
IgG $(\mathrm{g} / \mathrm{L})$ & -17.92 & $\leq 0.001$ & -66.13 & $\leq 0.001$ & $\leq 0.001$ \\
C3 $(\mathrm{g} / \mathrm{L})$ & -15.09 & $\leq 0.001$ & -22.79 & $\leq 0.001$ & $\leq 0.001$ \\
C4 $(\mathrm{g} / \mathrm{L})$ & -24.81 & $\leq 0.001$ & -30.30 & $\leq 0.001$ & $\leq 0.001$ \\
\hline
\end{tabular}

Note: ESR: erythrocyte sedimentation rate; CRP: C-reactive protein; IgA: immunoglobulin A; IgM: immunoglobulin M; IgG: immunoglobulin G; C3: complement C3; C4: complement $\mathrm{C} 4$. $Z_{0}$ represents the standardized test statistics before and after treatment in the control group. $P_{0}$ represents the comparison of the control group before and after treatment. $Z_{1}$ represents the standardized test statistics before and after treatment in the experimental group. $P_{1}$ represents the comparison of the experimental group before and after treatment. $P_{2}$ represents comparison between both groups after treatment.

4676 integrated evaluation records of IgA in the control group. The clinical significance is that every time the patient's comprehensive index improves by one point, they need to walk 17.33 steps, or, every step forward, the 
comprehensive improvement rate is $14.20 \%$. There are 4794 comprehensive evaluation records of $\operatorname{IgA}$ in the experimental group. The clinical significance is that every time the patient's comprehensive index improves by one point, they need to walk 12.05 steps, or, every step forward, the comprehensive improvement rate is $18.40 \%$. There were 4,675 and 4,800 integrated evaluation records for IgG in both groups. The patient improvement indexes in both groups were 0.179 and 0.204 . The clinical significance was that, for every increase in the integrated index, the patients had to walk 13.82 and 10.86 steps. There are 4670 integrated evaluation records for C3 in the control group. The clinical significance is that every time the comprehensive index of the patient improves by one point, they need to walk 15.17 steps, or, every step forward, the comprehensive improvement rate is $16.30 \%$. There are 4796 comprehensive evaluation records for patients in the treatment group's C3. The clinical significance is that every time the comprehensive index of the patient improves by one point, they need to walk 9.07 steps, or, every step forward, the comprehensive improvement rate is $24.40 \%$. There were 4,670 and 4,796 integrated evaluation records for $\mathrm{C} 4$ in both groups. The patient improvement indexes in both groups were 0.270 and 0.353. With regard to the clinical significance, for every increase in the integrated index, the patients had to walk 9.12 and 6.28 steps (Table 6 and Figure 4).

\section{Discussion and Conclusion}

Clustering is unsupervised learning and separated the data from groups (call as clusters) on account of their similar attributes. Apriori algorithm is a typical Boolean association rule frequent itemset algorithm [15]. The long-term correlation and mathematical probability theory of the random walking model are similar to the development of human diseases. Each symptom of the disease is affected by many factors such as the patient's self-perception, the body's ability to resist disease, and treatment measures. Whether the random walk model has long-term correlation, whose direct meaning is whether the index system is effective, is of great significance to the establishment of a comprehensive index system that is widely recognized and effective in Chinese medicine clinics.

$\mathrm{OA}$ is regarded as a kind of "arthralgia syndrome" in traditional Chinese medicine theory [16]. Traditional Chinese medicine treatment can effectively improve pain, dysfunction, and other symptoms and reduce the recurrence rate [17]. Herbal therapy is on the basis of the syndrome differentiation for the sake of satisfy personalized needs of different patients. Despite the use of single herb, physicians love herbal formulas, as well as complex mixtures of numerous herbs with abundant therapeutic compounds to maximize the curative effects and minimize the toxicity or adverse effects by interplay of different herbs [18]. As the painful obstruction, arthralgia syndrome signifies that either the limbs or the joints are subjected to pain and malfunction. The evil influence such as wind, cold, damp, and heat invades the physical organism, blocking the meridians and consuming the Promordial Qi and blood, which will make the joints painful, swollen, stiff and, in severe cases, deformed. Optimal administration of OA appeals for individual methods on the basis of various constitutions of human body. The individually integrated TCM approach in this research has been generated by clinical experience from the First Affiliated Hospital [19]. The approach comprises a fundamental treatment of orally administered Chinese herbal drugs and herbal patch on the basis of the severity of the patient symptoms.

Here, cluster and association rule analyses, graphical visualization of network diagram, propensity score methods, and random walking model data mining were applied to discriminate Chinese herbal medicine for OA treatment, compatible combinations, and significant therapeutic efficacy against $\mathrm{OA}$.

In the 13,083 Chinese herbal prescriptions, 426 types of herbs were applied to OA treatment. In the light of the nature, flavor, meridian tropism, and main efficacy of medicines, it was sensible to divide these into four categories tally with the principles of TCM, as shown in Table 1. The frequency of use of invigorating spleen for eliminating dampness herbs was 47,424 , which was the highest. The most commonly used single-flavor medicines are Poria, Flos Carthami, Radix Glycyrrhizae, Radix Salviae Miltiorrhizae, Pericarpium Citri Reticulatae, and so forth. Classified according to five flavors, sweetness and bitterness are the two most frequently used. Within the four classes of Chinese herbal medicine, the spleen meridian was used 44,470 times, sweet taste was applied 59,458 times, and bitter taste was applied 69,650 times. Sweet taste can replenish energy and tonify the spleen, while bitter taste is used to dispel dampness, on account of traditional Chinese medicine ideology. Both can verify that OA pathogenesis originates mainly from spleen deficiency and dampness. The development of muscle and its function are closely related to the function of spleen transportation. Based on modern pharmacology study, spleen-invigorating Chinese medicine can increase immune capacity, improve learning and memory function, and control the endocrine function of patients with impaired disease [20]. Through data mining technology, the high-frequency herbs and Chinese herbs compatibility for the treatment of osteoarthritis are summarized, and the drug characteristics and prescription rules of osteoarthritis are scientifically and objectively revealed. At length, we come to the conclusion that the regularity of traditional Chinese medicine in the treatment of osteoarthritis is invigorating spleen for removing dampness, promoting blood circulation and removing blood stasis, expelling wind and removing dampness, and clearing away heat and toxic materials, which can be used for reference in the treatment of clinical osteoarthritis.

Through cluster analysis, we found that high-frequency Chinese medicine combinations commonly used in this disease can be divided into four categories (Figure 2). The first group of Chinese medicines is to invigorate the spleen, dispel dampness, and promote blood circulation. The second kind of Chinese medicine expels wind and dredges collaterals. The third kind of Chinese medicine clears away heat 
TABLE 6: Random walking model of immune-inflammatory indices.

\begin{tabular}{|c|c|c|c|c|c|c|c|}
\hline Index & Group & $\begin{array}{l}\text { Maximum } \\
\text { random } \\
\text { fluctuation }\end{array}$ & $\begin{array}{l}\text { Walking positive } \\
\text { growth rate }\end{array}$ & $\begin{array}{l}\text { Random fluctuation } \\
\text { power law value }\end{array}$ & $\begin{array}{l}\text { Improvement } \\
\text { index }\end{array}$ & $\begin{array}{l}\text { Comprehensive } \\
\text { evaluation records }\end{array}$ & Ratio \\
\hline \multirow[b]{2}{*}{ ESR } & \multirow{2}{*}{$\begin{array}{c}\text { Control group } \\
\text { Experimental } \\
\text { group }\end{array}$} & 1410 & 0.108 & $0.448 \pm 0.099$ & 0.226 & 6253 & 9.26 \\
\hline & & 2494 & 0.196 & $0.520 \pm 0.126$ & 0.362 & 6883 & 5.11 \\
\hline \multirow[b]{2}{*}{ CRP } & \multirow{2}{*}{$\begin{array}{c}\text { Control group } \\
\text { Experimental } \\
\text { group }\end{array}$} & 1915 & 0.137 & $0.494 \pm 0.106$ & 0.276 & 6933 & 7.28 \\
\hline & & 3236 & 0.239 & $0.566 \pm 0.141$ & 0.426 & 7603 & 4.18 \\
\hline \multirow[b]{2}{*}{$\operatorname{IgA}$} & \multirow{2}{*}{$\begin{array}{l}\text { Control group } \\
\text { Experimental } \\
\text { group }\end{array}$} & 666 & 0.058 & $0.400 \pm 0.091$ & 0.142 & 4676 & 17.33 \\
\hline & & 882 & 0.083 & $0.512 \pm 0.135$ & 0.184 & 4794 & 12.05 \\
\hline \multirow[b]{2}{*}{$\operatorname{IgG}$} & \multirow{2}{*}{$\begin{array}{l}\text { Control group } \\
\text { Experimental } \\
\text { group }\end{array}$} & 835 & 0.072 & $0.407 \pm 0.095$ & 0.179 & 4675 & 13.82 \\
\hline & & 979 & 0.092 & $0.463 \pm 0.123$ & 0.204 & 4800 & 10.86 \\
\hline \multirow[b]{2}{*}{ C3 } & \multirow{2}{*}{$\begin{array}{c}\text { Control group } \\
\text { Experimental } \\
\text { group }\end{array}$} & 760 & 0.066 & $0.397 \pm 0.091$ & 0.163 & 4670 & 15.17 \\
\hline & & 1172 & 0.110 & $0.515 \pm 0.128$ & 0.244 & 4796 & 9.07 \\
\hline \multirow[b]{2}{*}{$\mathrm{C} 4$} & \multirow{2}{*}{$\begin{array}{l}\text { Control group } \\
\text { Experimental } \\
\text { group }\end{array}$} & 1265 & 0.110 & $0.484 \pm 0.127$ & 0.270 & 4670 & 9.12 \\
\hline & & 1693 & 0.159 & $0.534 \pm 0.121$ & 0.353 & 4796 & 6.28 \\
\hline
\end{tabular}

Note: ESR: erythrocyte sedimentation rate; CRP: C-reactive protein; IgA: immunoglobulin A; IgG: immunoglobulin G; C3: complement C3; C4: complement C4.

and detoxifies. The fourth kind of traditional Chinese medicine is to clear away heat and detoxify and relieve collaterals.

According to the set of principles for drug association analysis, there were a total of 11 rules involving couplet medicines resulting. There are some interesting association rules that are usually used by ancient and modern physicians for combinations of couplet medicines. The couplet medicine of invigorating the spleen medicinal is Pericarpium Citri Reticulata-Poria and Radix Glycyrrhizae-Poria, while bloodactivating medicinal is Radix Salviae Miltiorrhizae-Flos Carthami and Flos Carthami-Semen Persicae, for instance. We find that the obtained association rules invigorating the spleen, dispelling dampness, promoting blood circulation, and clearing away heat and detoxifying treatments have concomitant uses. Based on the relationship rule mining, synergistic herbal groups could be derived; nevertheless, it is necessary to further analyze the associated mechanism (Table 2 and Figure 3). At the same time, there is a strong correlation between the compatibility of traditional herbal medicine and the optimization of immune-inflammatory indices (Table 3). Herba Taraxaci Mongolici, Flos Carthami, and Semen Coicis are related to inflammatory index, such as ESR and CRP. The new research demonstrated that taraxasterol has the in vitro anti-inflammatory effect, which is one of the chief active components isolated from Herba Taraxaci Mongolici [21]. Flos Carthami [22] and Semen Coicis [23] are perhaps connected to its antioxidation and anti-inflammatory properties. Semen Persicae, Poria, Radix Glycyrrhizae, and Radix Salviae Miltiorrhizae are related to immune index, for instance, IgM, IgA, and IgG. Radix Glycyrrhizae and Pericarpium Citri Reticulatae are bounded up with C4 and C3. Poria
[24] is commonly used as a tonic and antiaging traditional Chinese medicine, which is traditionally used in combination with other TCM to enhance immunity. Radix Glycyrrhizae Polysaccharide, one of the chief bioactive components in Radix Glycyrrhizae, has been reported to participate in regulation of immunity and phagocytosis, as well as anticomplement [25]. Volatile oils and flavonoids in Pericarpium Citri Reticulatae are considered to be major components, which act alone or jointly to combat inflammatory responses and lipid peroxidation, followed by attenuating immunological reaction [26]. To conclude, the therapeutic effects resulting from combinations of couplet medicines are quite different.

The generation of Chinese formulas with various herbs is not random. In this research, data mining technology was used to determine the rules for the application of Chinese herbal medicine in OA treatment in our hospital, in order to test the efficacy of Chinese herbal medicine in OA treatment. As a convenient prescription hospital preparation of Anhui Traditional Chinese Medicine Hospital, Huangqin Qingre Chubi capsule (Anhui medicine No. ZL201110095718.X) consists of Radix Scutellariae Baicalensis, Radix et Rhizoma Clematidis Chinensis, Semen Coicis, and Semen Persicae. It has the function of invigorating spleen and removing dampness and clearing heat and removing arthralgia with long history in clinical OA treatment. As a prescription preparation of our hospital, Furong ointment has the effect of clearing heat, detoxifying swelling, cooling blood, and relieving pain.

The variations in the values of immune-inflammatory indices were analyzed to evaluate the efficacy. Before that, we used propensity scoring method to match the baseline characteristics and immune-inflammatory indices of the two 

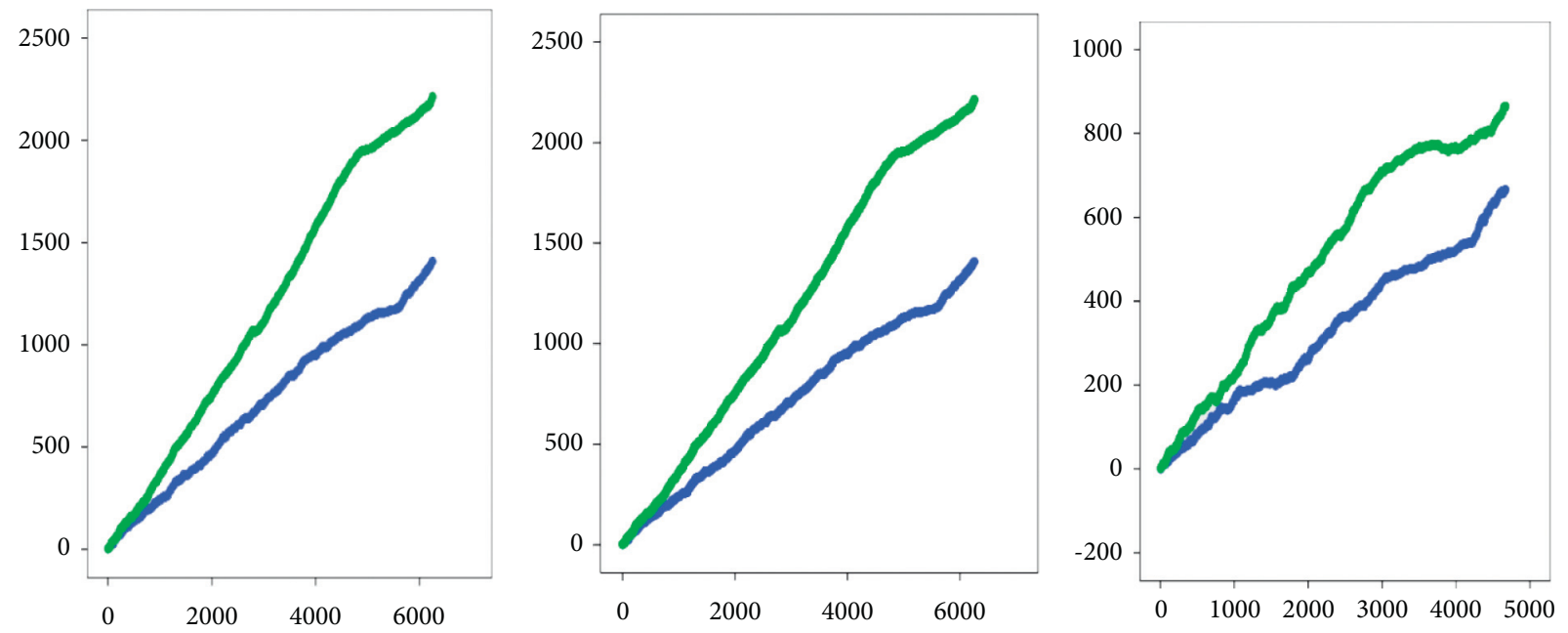

- Control group ESR

- Experimental group ESR
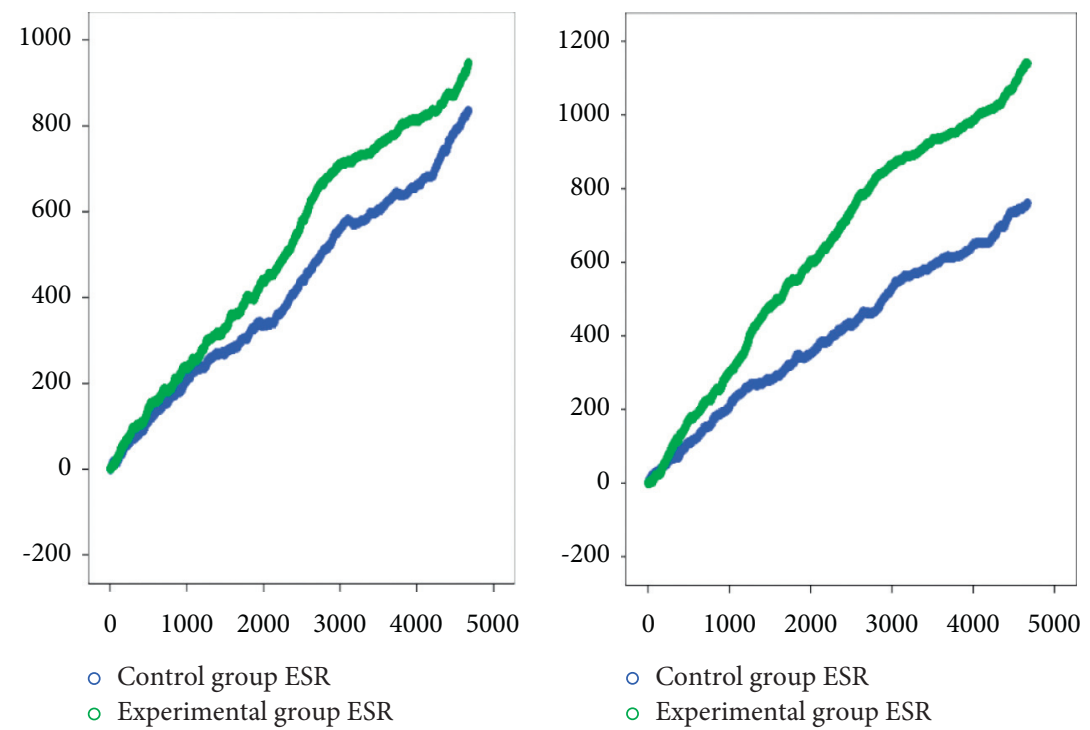

- Control group ESR

- Experimental group ESR

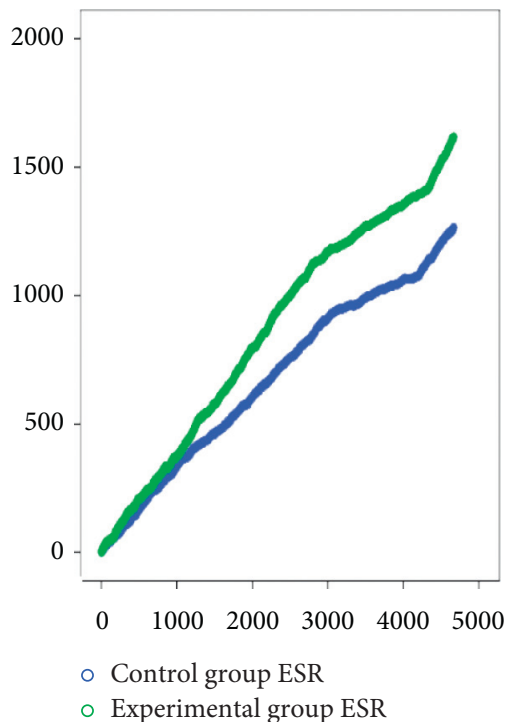

FIGURE 4: Random walking model of immune-inflammatory indices in OA patients. Note: green line represents the experimental group. Blue line represents the control group. The length of the horizontal line increases with the increase in the number of walking steps. The height of the vertical line increases with intervention efficacy and response.

groups to reduce research bias. As you can see, the propensity score matching resulted in 4438 pairs of patients from the experimental group and the control group. Unlike the results before adopting propensity score matching, there were no significant differences between both groups in terms of age, sex, BMI, LOS, and underlying diseases (coronary heart disease, hypertension, cerebral infarction, diabetes, and osteoporosis) (Table 4$)(P>0.05)$. However, inevitable imbalance persisted in chronic gastritis, anemia, and fatty liver. After applying propensity score matching, the immune-inflammatory indices were balanced between the two groups, and there was no significant difference $(P>0.05)$ (Table 4). According to the distribution rules, there were 7,119 patients in the control group and 5,964 patients in the experimental group. The statistical results show that, compared with single drug, the drug combination improved ESR, C3, IgA, CRP, IgG, and C4 more effectively (Table 5).
Therefore, strong therapeutic efficacy of Chinese herbal decoctions integrated with prescription preparations was found. A random walking model was applied to assess the immune-inflammatory indices of both groups of OA patients. The patient's immune-inflammatory indices refer to the long-term relationship between the changes of ESR, IgA, IgG, C3, CRP, and C4 and the intervention measures the patient receives, which means that the treatment measures the patient receives affect the changes of the patient's immune inflammation index. The treatment group's ESR, IgA, CRP, C3, C4, and IgG are better than the control group's in terms of the maximum random fluctuation, the positive growth rate of walking, the increase rate of the comprehensive evaluation index, the comprehensive improvement rate, the number of records of the comprehensive evaluation index, and the expected improvement value. But the improvement of IgM is not obvious. There is a long-term 
correlation between the comprehensive evaluation indexes and intervention measures of the two groups of patients, and the improvement effect of indexes of the experimental group is better than that of the control group (Table 6 and Figure 4).

The Chinese medicine is through the classification of the causes of OA, symptomatic administration, and the use of appropriate methods of treatment [27]. As for the diagnosis and treatment model based on diagnostic personality and diagnostic integration, TCM medical records provide good evidence for TCM evidence-based practice, which reflects the comprehensive application of TCM principles, methods, formulas, and drugs rather than the comprehensive application of TCM. It is not only the true record of medical activities but also the reflection of the physician's clinical experience and thought process. Data mining, also known as knowledge discovery, is the task of discovering laws or patterns hidden in a large amount of data, which can bring improvement and further development of TCM academic technology [28]. However, Chinese medicine discusses and studies human body from the whole and function level, while data mining based on data induction and machine learning is limited to exploring the surface statistical law and lacks the discussion on the internal mechanism of the system. On the premise of guaranteeing the integrity of the data, therapeutic efficacy is difficult to be fully verified because of the complexity of the inpatient medical records, including the incomplete and unquantifiable information in the data. Beyond that, Chinese medicine studies human body from the whole and function level, but the data mining based on data induction and machine learning is limited to exploring the surface statistical law and lacks the discussion of the internal mechanism of the system.

In conclusion, we built a random walking model, which is based on laboratory indicators and combined with cluster analysis, association rules, graphical visualization of network diagram, and propensity score methods. We found that Chinese medicine compatibility can improve the immune-inflammatory indices of patients suffering from OA. Different groups of these drugs bring about various effects in OA treatment, which provide experience for clinical treatment of OA.

\section{Data Availability}

The datasets generated for this study are available upon request from the corresponding authors.

\section{Conflicts of Interest}

The authors declare that the research was conducted in the absence of any commercial or financial relationships that could be construed as potential conflicts of interest.

\section{Authors' Contributions}

Qiao Zhou performed the statistical analysis and drafted the manuscript. Jian Liu explained and revised the data in the manuscript. Ling Xin provided technical knowledge. Yanyan Fang, Lei Wan, Dan Huang, Jinchen Guo, and Jianting Wen collected the data required for this manuscript. All the authors read and approved the final version of the manuscript.

\section{Acknowledgments}

The authors would like to thank Yanyan Fang and Dan Huang for providing the basis for the manuscript. This study was financially supported by the Anhui Key Research and Development Program Foreign Science and Technology Cooperation Project (no. 201904b11020011), the Study on Individualized Treatment of Osteoarthritis with Integrated Traditional Chinese and Western Medicine Based on Clinical Big Data (no. KJ2019A0439), the Ministry of Science and Technology National Key Research and Development Program Chinese Medicine Modernization Research Key Project (no. 2018YFC1705204), the National Nature Fund Program (no. 81973655), the Anhui Provincial Quality Engineering Teaching and Research Project (no. 2018jyxm1068), the Anhui Famous Traditional Chinese Medicine Liu Jian Studio Construction Project (Traditional Chinese Medicine Development Secret [2018] no. 11), the National Key Innovative Talents Training Project of Traditional Chinese Medicine (National Education Letter of Traditional Chinese Medicine [2019] no. 128), the Key Research and Development Plan Project of Anhui Province (no. 201904a07020004), the Anhui Provincial Laboratory of Applied Basis and Development of Internal Medicine of Modern Traditional Chinese Medicine (no. 2016080503B041), and the 12th Batch of "115" Innovation Team of Anhui Province (Anhui Talent Office [2019] no. 1).

\section{References}

[1] X. Song, Z. Wang, P. Zhang, M. Zhao, L. Yang, and W. Zhang, "A comparison of the efficacy and safety of traditional Chinese medicine external treatment for the knee osteoarthritis," Medicine, vol. 100, no. 1, Article ID e24115, 2021.

[2] R. F. Loeser, S. R. Goldring, C. R. Scanzello, and M. B. Goldring, "Osteoarthritis: a disease of the joint as an organ," Arthritis \& Rheumatism, vol. 64, no. 6, pp. 1697-1707, 2012.

[3] B. McCarberg and P. Tenzer, "Complexities in the pharmacologic management of osteoarthritis pain," Current Medical Research and Opinion, vol. 29, no. 5, pp. 539-548, 2013.

[4] B. Chen, H. Zhan, J. Marszalek et al., "Traditional Chinese medications for knee osteoarthritis pain: a meta-analysis of randomized controlled trials," The American Journal of Chinese Medicine, vol. 44, no. 4, pp. 677-703, 2016.

[5] S. I. Lee, H. Ghasemzadeh, B. Mortazavi et al., "Remote patient monitoring: what impact can data analytics have on cost?" in Proceedings of the 4th Conference on Wireless Health, Baltimore MD. USA, November 2013.

[6] A. Garcia-Rudolph, A. G. Molina, E. Opisso, and J. M. Tormos, "Cluster analysis for personalized web-based cognitive rehabilitation treatments in traumatic brain injury," JMIR Medical Informatics, vol. 8, no. 10, Article ID e16077, 2019.

[7] L. Chen, Y. Pan, Y. Chen, and X. Xu, "Efficient parallel algorithms for Euclidean distance transform," The Computer Journal, vol. 47, no. 6, pp. 694-700, 2004.

[8] S. Raj, D. Ramesh, and K. K. Sethi, "A spark-based apriori algorithm with reduced shuffle overhead," The Journal of Supercomputing, vol. 77, no. 1, pp. 133-151, 2020. 
[9] A. Derouiche, A. Layeb, and Z. Habbas, "Mining interesting association rules with a modified genetic algorithm," Pattern Recognition and Artificial Intelligence, vol. 1322, pp. 274-285, 2021.

[10] L. Ding, S. Xie, S. Zhang et al., "Delayed comparison and apriori algorithm (DCAA): a tool for discovering proteinprotein interactions from time-series phosphoproteomic data," Frontiers in Molecular Biosciences, vol. 7, Article ID 606570, 2020.

[11] J. Y. Yang, M. Webster-Clark, J. L. Lund, R. S. Sandler, E. S. Dellon, and T. Stürmer, "Propensity score methods to control for confounding in observational cohort studies: a statistical primer and application to endoscopy research," Gastrointestinal Endoscopy, vol. 90, no. 3, pp. 360-369, 2019.

[12] R. Hanaya, C. Victoria, C. Susan, E. Williamson, and S. Cro, "An evaluation of inverse probability weighting using the propensity score for baseline covariate adjustment in smaller population randomised controlled trials with a continuous outcome," BMC Medical Research Methodology, vol. 20, no. 7, p. 70, 2020.

[13] K. Pearson, "The problem of the random walk," Nature, vol. 72 , no. 1867 , p. 342, 1905.

[14] C.-K. Peng, S. V. Buldyrev, A. L. Goldberger et al., "Longrange correlations in nucleotide sequences," Nature, vol. 356, no. 6365, pp. 168-170, 1992.

[15] J. Kim, U. Yun, E. Yoon, J. C.-W. Lin, and P. Fournier-Viger, "One scan based high average-utility pattern mining in static and dynamic databases," Future Generation Computer Systems, vol. 111, pp. 143-158, 2020.

[16] H. Huang, Z. F. Zhang, F. W. Qin et al., "Icariin inhibits chondrocyte apoptosis and angiogenesis by regulating the TDP -43 signaling pathway," Molecular Genetics \& Genomic Medicine, vol. 7, no. 4, Article ID e00586, 2019.

[17] L. Wang, X.-F. Zhang, X. Zhang et al., "Evaluation of the therapeutic effect of traditional Chinese medicine on osteoarthritis: a systematic review and meta-analysis," Pain Research and Management, vol. 2020, Article ID 5712187, 23 pages, 2020.

[18] M. Wang, L. Liu, C. S. Zhang et al., "Mechanism of traditional Chinese medicine in treating knee osteoarthritis," Journal of Pain Research, vol. 13, pp. 1421-1429, 2020.

[19] Y. Fang, J. Liu, L. Xin et al., "Identifying compound effect of drugs on rheumatoid arthritis treatment based on the association rule and a random walking-based model," BioMed Research International, vol. 2020, Article ID 4031015, 10 pages, 2020.

[20] X. Zhang, L. Li, T. Chen et al., "Research progress in the effect of traditional Chinese medicine for invigoration on neurotransmitter related diseases," Evidence-Based Complementary and Alternative Medicine, vol. 2018, Article ID 4642018, 14 pages, 2018.

[21] Y. Wang, G. H. Li, X. Y. Liu, L. Xu, S.-S. Wang, and X.-M. Zhang, "In vivo anti-inflammatory effects of taraxasterol against animal models," African Journal of Traditional, Complementary and Alternative Medicines, vol. 14, no. 1, pp. 43-51, 2017.

[22] P. K. Fu, T. L. Pan, C. Y. Yang, K. C. Jeng, N. Y. Tang, and C. L. Hsieh, "Carthamus tinctorius L. ameliorates brain injury followed by cerebral ischemia-reperfusion in rats by antioxidative and anti-inflammatory mechanisms," Iranian Journal of Basic Medical Sciences, vol. 19, no. 12, pp. 13681375, 2016.

[23] J. Du, G. Yin, Y. Hu et al., "Coicis semen protects against focal cerebral ischemia-reperfusion injury by inhibiting oxidative stress and promoting angiogenesis via the TGF $\beta /$ ALK1/ Smad1/5 signaling pathway," Aging, vol. 12, no. 1, 2020.

[24] C.-L. Chao, H.-W. Huang, M.-H. Su, H.-C. Lin, and W.-M. Wu, "The lanostane triterpenoids in poria cocos play beneficial roles in immunoregulatory activity," Life, vol. 11, no. 2, p. 111, 2021.

[25] X. Li, X. He, B. Liu et al., "Maturation of murine bone marrow-derived dendritic cells induced by radix glycyrrhizae polysaccharide," Molecules, vol. 17, no. 6, pp. 6557-6568, 2012.

[26] J. Wu, X. Ye, S. Yang, H. Yu, L. Zhong, and Q. Gong, "Systems pharmacology study of the anti-liver injury mechanism of citri reticulatae pericarpium," Frontiers in Pharmacology, vol. 12, 2021.

[27] H. E. Jun-Lei, R. Z. Zhang, and Z. L. Chen, "Clinical observation of juanbi decoction combined with joint mobilization on treatment of knee osteoarthritis," Chinese Journal of Experimental Traditional Medical Formulae, vol. 22, Article ID 168171, 2016.

[28] X. Y. Dong, S. Qi, and M. Jiang, "Application of data mining in the research of traditional Chinese medicine," Global Traditional Chinese Medicine, vol. 10, pp. 364-368, 2017. 